\title{
Measuring Greenery Supportiveness in Park for Physical Activity Using Smartphone Panoramic Image and a Green Vegetation Extraction Index
}

\author{
Xinyang Yu \\ College of Resources and Environment, Shandong Agricultural University \\ Wenqian Huo ( $\nabla$ wq_huo@163.com) \\ UF/IFAAS Extension \\ Beibei Niu \\ College of Resources and Environment, Shandong Agricultural University \\ Maosen Zhao \\ Information Center, Taishan Sanatorium of Shandong Province
}

\section{Research}

Keywords: visible greenery, green vegetation extraction index, iPhone, panoramic image, Googlestreetview

Posted Date: July 23rd, 2020

DOI: https://doi.org/10.21203/rs.3.rs-45628/v1

License: (c) This work is licensed under a Creative Commons Attribution 4.0 International License.

Read Full License 


\title{
1 Measuring greenery supportiveness in park for physical activity using 2 smartphone panoramic image and a green vegetation extraction index
}

* Correspondence: wq_huo@163.com

\begin{abstract}
:
Physical activity in urban park contributes substantial health benefits for people. However, due to the absence of profile image systems, objective assessment of greenery supportiveness in park from people's view is rare or even nonexistent. Manager and planner's ability to manage and plan park landscapes effectively and efficiently is, therefore, limited. Typical smartphone, such as iPhone, has been widespread used to facilitate daily life. Image captured using panoramic mode in smartphone may be an alternative that can provide profile views of park landscape and greenery, yet no research seems available in literature. We investigated the feasibility of typical smartphone captured panoramic image and proposed a green vegetation extraction index (GVEI) for quantitatively depicting and monitoring the park greenery. A five-kilometer loop road network in Homestead, Florida, whose length and width are similar to the park trail, was selected as the test bed. The iPhone Xs was operated to take panoramic images of one hundred randomly selected investigation sites. Google Street View panoramic images acquired in the identical sites were downloaded and processed as comparison. The results demonstrated that the smartphone panoramic image is time-sensitive and well suited for assessing the trail-level greenery. The GVEI is an objective measurement indicator of trailside greenery. The smartphone panoramic image in combination with GVEI can be used to better understand the greenery supportiveness for physical activity and guide greenery planning in urban park.
\end{abstract}

Key words: visible greenery; green vegetation extraction index; iPhone; panoramic image; Google street view 


\section{Introduction}

Physical activities, e.g. walking, jogging and running, are widely affirmed as effective ways associated with adults' and adolescents' health benefits [1,2]. Empirical studies have demonstrated that physical activity in green space such as treelined street and urban park could yield more physiological and psychological health benefits above in other environments [3-5]. For instance, psychological responses to walking through green space showed significantly correlation with trait anxiety levels [6]. Walking in parks leads to physiological, psychological relaxation, and varied landscape appreciation of mid-aged and old people [7]. Adolescent girls live near more parks with amenities that are conducive to walking and with active features, engage in more moderate or vigorous physical activity than those with fewer parks [8]. People visiting parks were more likely to achieve the recommended levels of physical activity than those ignored using parks [9].

We know that $90 \%$ of the information that people receive in the environment comes from vision sensory function [10]. The greenery makes important contributes to improve the attractive and favorability of street and park. However, understanding the sensory functions of greenery is restrained by the difficulty of assessing the visual quality of greenery on residents. Traditional methods used to measure residents' perceptions of profile views of urban greenery include questionnaire survey, field audits and remotely sensed (RS) extraction data. These methods have merits and limitations. The questionnaire survey method could get first-hand information, while it may evoke concerns over response bias [11]. Field audit method is more objective though raters have to visit all the field locations [12]. RS data, such as tree count or vegetation fraction estimation, is more objective and time-efficient. However, such data are mainly acquired from overlooking view, which is different to that of resident. Besides, the absence of penetration ability limits the ability of current RS images to detect multi-layer greenery such as shrubs and lawns under canopies [13].

Street greenery studies have examined profile image systems and developed quantitative depicting methods to address these limitations and objective monitoring greenery. A green view index (GVI) was introduced to indicate the greenery that resident can see on street-side [14]. In the process of GVI-based images acquisition, trained researchers will stand on the set locations and use film or digital camera to take the pictures of the street side at different horizontal directions and a vertical altitude of $1.6 \mathrm{~m}$ [10]. New profile image system, such as Google Street View (GSV), has uploaded and renewed images periodically for public free download and application. These images were captured by dome cameras on top of special vehicles driving along the street with centimeter spatial resolution and had view angles similar to those of residents [15]. Based on the GSV imagery, Yang et al. [16] estimated the visibility of urban street greenery using a street GVI and four pictures taken in four horizontal directions (north, south, east, and west) to evaluate the street greenery in the study area, which was further applied in other typical cities, e.g. Hongkong [17] and Singapore [18]. Li et al. modified the process of Google Street View images acquisition to more accurately assess the profile of street-level greenery. The results all found that GSV images were qualified and efficient measurements of street-level greenery [13].

Though GSV imagery can be used to monitor the street-side greenery, it is not available in park because the width of the park trail could not accommodate the capturing vehicle. Thus, greenery study in urban park is limited in empirical study due to the absence of reliable profile image dataset. Recently, iPhone series smartphone gradually gains popularity [19, 20]. The report from ComScore (https://www.comscore.com/Insights/Rankings\#tab mobile iPhone oems/) found that iPhone ranks as the top original equipment manufacturer (OEM) with 51.2\% of market share on April 2020 in the US. Compared to film and digital cameras, iPhone is more portable and has professional parameter settings to take panoramic images, which may be a feasible alternative of GSV images for park greenery monitoring. This study thus strived to 
examine the performance of iPhone panoramic image (iPhone image hereafter) in green vegetation extraction and GVEI computation.

\section{Study region}

A five-kilometer long and two-lane road network including SW 272th Street (North Road), SW 280th Street (South Road), SW 187 ${ }^{\text {th }}$ Avenues (East Road) and SW 197 ${ }^{\text {th }}$ Avenue (West Road) in Homestead, Miami-Dade County, was selected to conduct the study (Figure 1). The road network was delineated based on visual interpretation and reference from the Open Street Map (OSM, openstreetmap.org). The park trail-like road network and similar evergreen vegetation coverage rates make the study region ideal for the examination.

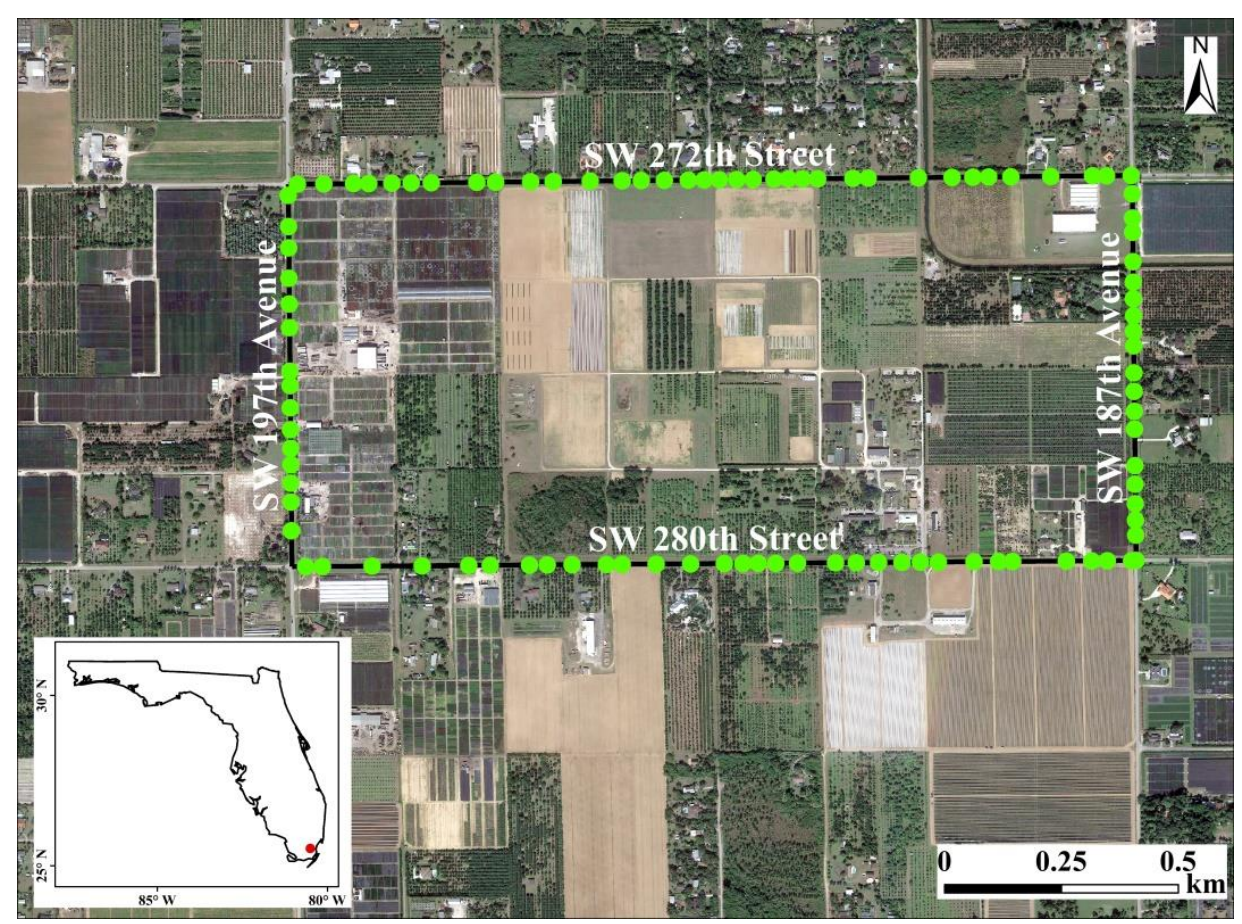

Figure 1. Location of the study region. The green dots denote the investigation sites where high quality panoramic image were obtained to extract the greenery. The background image was obtained from Google map.

\section{Data and methods}

\section{Data acquisition and processing}

One hundred investigation sites were randomly generated using the geographical analytic and statistics software in the road network. The distance between two adjacent sample sites was set to fifty meters and the minimum distance thirty meters. It must be worth noting that the term "greenery" is consistently used to describe the level of green vegetation due to vegetation (or chlorophyll) and differentiate it from "greenness" of the tasseled cap transformation in conventional remote sensing. The geographical location of the investigation sites were obtained and encoded with sequential ID (0-99) for greenery investigation.

To obtain iPhone image, we walked from the location of ID 0 and headed to the direction of ID 99 on March 15, 2020. At each investigation site, we held the iPhone Xs at a zero vertical angle to take panoramic images at an altitude of $1.6 \mathrm{~m}$, which was exactly the common eye's height and angle of resident taking physical activity. Because the panoramic image in iPhone Xs covers a horizontal scope of about $270^{\circ}$, we took two images for each investigation site to cover the whole 
horizontal visual landscape. After capturing the panoramic images of all the sites, the images at each site were stitched to obtain one integrated and seamless panoramic image. Thus, one hundred panoramic images were finally obtained for further processing and analysis.

As GSV images have been found to be suited for urban street greenery investigation, the GSV panoramic images for the study sites at zero vertical angle (GSV images hereafter) were downloaded via GSV image API as comparison [16]. Among the one hundred investigation sites, GSV images of twenty-nine sites were captured in 2011, and the others were obtained in 2019.

\section{3}

114

\section{Green vegetation extraction and accuracy verification}

Vegetation index commonly used in multi-spectral remotely sensed image analysis, such as Normalized Difference Vegetation Index (NDVI), employs the information of near infrared (NIR) and red band information [21,22]. High reflectance at the NIR band and high absorption at the red band make green vegetation relatively easy to be distinguished from images. However, both iPhone and GSV images do not include NIR information but captured only red, green and blue band information, and the spectral ranges of the common features existing in urban park, e.g. sky, greenery shade, trunk, bare soil, facade of building, and pavement, largely overlap (Figure 2a). Such limitation makes it difficult to identify vegetation and its coverage in the original panoramic images.
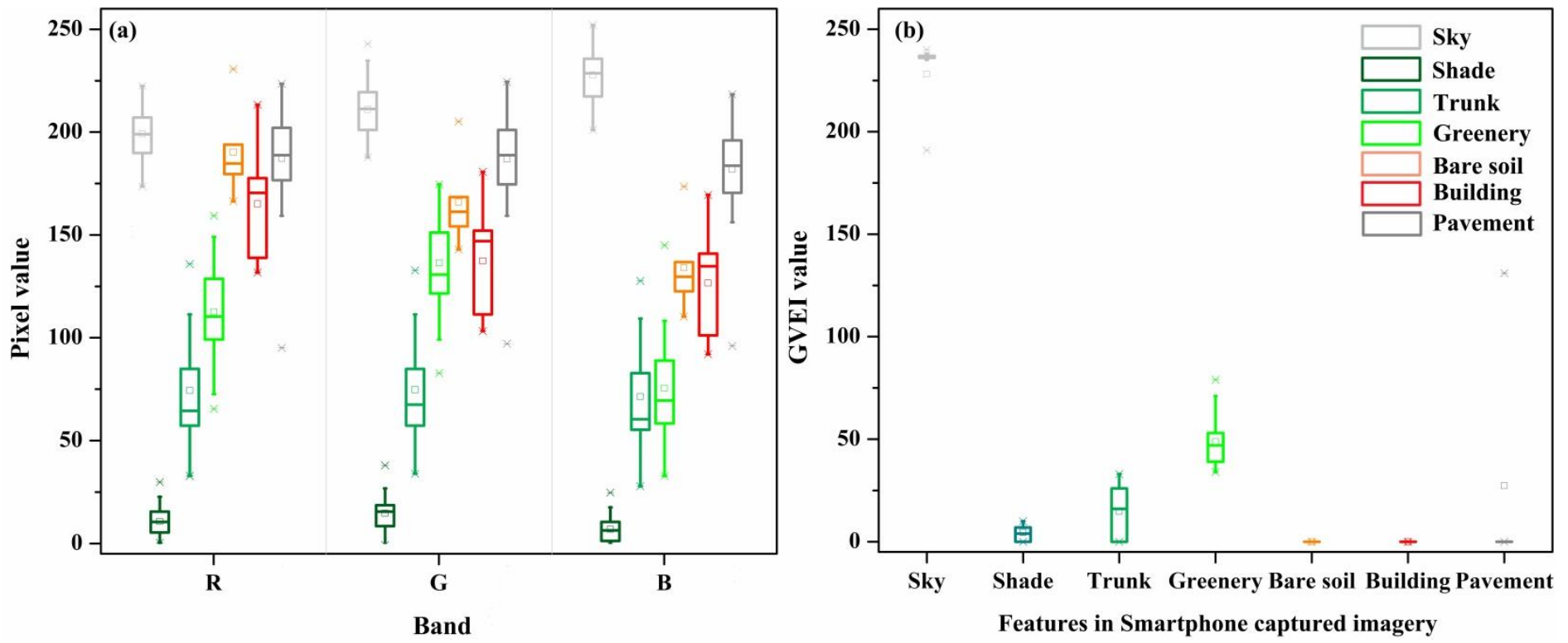

Figure 2. Pixel value and GVEI range of common features in the iPhone captured panoramic imagery. (a): Pixel value range of common features; (b): GVEI ranges of the features.

The lack of NIR information in the panoramic images stimulated us to use vegetation indices that do not employ NIR information in this study. We proposed to use the Green Vegetation Extraction Index (GVEI, Eq. 1) to detect green vegetation.

$\mathrm{GVEI}=\frac{G}{R * B}$

where G, R and B represents the green, red and blue band value of iPhone image, respectively. 
After the GVEI grey scale images were obtained using the above equation, the decision tree method was used to distinguish greenery by specifying its GVEI value ranges (Figure 2b). To examine the greenery identification accuracy,

Table 1. Criteria GVI derived system of vegetation distribution in the study site.

\begin{tabular}{ccl}
\hline Level & Vegetation distribution index $(\%)$ & \multicolumn{1}{c}{ Vegetation distribution pattern } \\
\hline Grade 1 & $<10$ & $\begin{array}{l}\text { Null or very small proportion of green vegetation in the BSV image; Strong } \\
\text { perspective of manmade landscape } \\
\text { Relative low proportion of green vegetation; Have impression of manmade } \\
\text { landscape } \\
\text { Grade } 2\end{array}$ \\
Grade 3 & $10-20$ & $\begin{array}{l}\text { Moderate percentage of vegetation coverage; The artificial environment is } \\
\text { natural and comfortable }\end{array}$ \\
Grade 4 & $>30$ & $\begin{array}{l}\text { High percent of vegetation coverage; Strong visual and spiritual pleasure, most } \\
\text { beneficial to human health }\end{array}$ \\
\hline
\end{tabular}
were summarized into four grades using the vegetation distribution values with an interval of 10\% (Table 1). the supervised artificial neural network classifier (ANNc) was employed to distinguish the green vegetation pixels in the iPhone images, and manual classification results based on visual refinement of ANNc results were used as truth reference data. Inspired by the biological nervous system, ANNc is composed of an interconnected neurons network. The learning process of ANNc is to adjust the connection weights between the neurons. Each neuron forms a node in the whole network and after training each node is assigned with a determined threshold. For each interconnection between two nodes, a weight is assigned to represent the link-strength between the neurons [23]. Theoretically, ANNc is able to achieve an accurate result with high generalization capacity. It was found to outperform other supervised classifiers when classifying multi-spectral RS images [24]. The confusion matrix was computed using fifty randomly selected panoramic images. Four coefficients (user's accuracy, producer's accuracy, overall accuracy and Kappa coefficient) were computed by comparing the GVEI and ANNc results with manual depiction images.

\section{Vegetation distribution and gradation}

The panoramic green view index was used to represent the greenery in each study sites. It was calculated using the proportion of green vegetation pixels in the panoramic image.

Panoramic green view index $=\frac{N_{\text {veg }}}{N_{\text {total }}} * 100$

where $N_{\text {veg }}$ is the pixel amount of green vegetation, and $N_{\text {total }}$ represents the total pixels of iPhone and GSV images. Based on the landscape layout in the study site and the gradation system of previous studies [14,25], the investigation sites

\section{Results and discussion}

\section{Green vegetation extraction}

The green vegetation classification accuracies of selected images using confusion matrix indicated that the overall (kappa) accuracy values were higher than $0.90(0.85)$, and both user's and producer's accuracies of vegetation were greater than 0.85, indicating the green vegetation identification from the iPhone images using GVEI method were reliable and could be used for greenery distribution analysis (Table 2). The green vegetation extraction of ANNs underperformed GVEI method, the overall accuracy and Kappa coefficient were also much low than those of GVEI method. 
Table 2. Accuracy assessment of green vegetation using GVEI method and ANNc.

\begin{tabular}{clll}
\hline \multirow{2}{*}{ Feature } & Accuracy & \multicolumn{2}{l}{ iPhone image classification method } \\
\cline { 3 - 4 } & & GVEI & ANNc \\
\hline \multirow{2}{*}{ Green vegetation } & User's Accuracy & $0.98-1.00$ & $0.86-0.89$ \\
\cline { 3 - 4 } & Producer's Accuracy & $0.92-0.95$ & $0.84-0.86$ \\
\hline \multirow{2}{*}{ Others } & User's Accuracy & $0.89-0.94$ & $0.61-0.84$ \\
\cline { 3 - 4 } & Producer's Accuracy & $0.85-0.89$ & $0.54-0.76$ \\
\hline All & Overall Accuracy & $0.92-0.95$ & $0.78-0.80$ \\
\cline { 3 - 4 } All & Kappa Coefficient & $0.87-0.90$ & $0.74-0.86$ \\
\hline
\end{tabular}

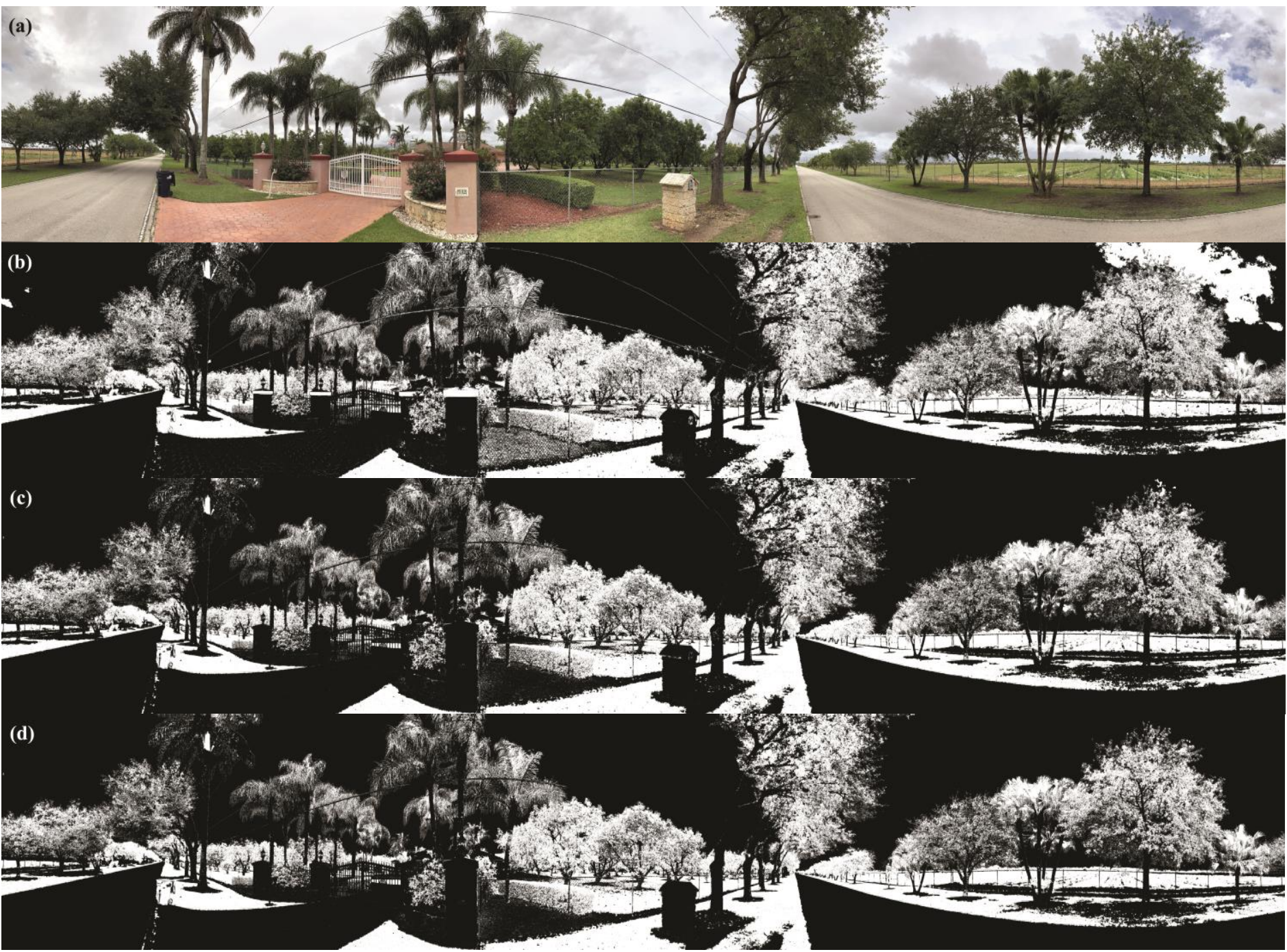

Figure 3. An example of iPhone image and green vegetation classification results. (a): Original image, (b): ANNc result, (c): GVEI processing result, and (d): manual classification.

To exhibit the classification results more vividly, the investigation site-ID 57 was selected because of its most complicated layout pattern in the study region. The original panoramic image, processing results using ANNc, GVEI and manual classification methods were showed in Figure 3, respectively. Comparison could find that ANNc complicated green vegetation with some shade, bare soil and wire in the central part of the image, and sky features in the right edge of the image (Figure 3b), GVEI method provided similar green vegetation classification results to manual classification method (Figures 3c and 3d). The classification results using GVEI were much better than that based on ANNc. To further quantitatively assess the classification accuracy, the vegetation distributions of images classified using the GVEI and manual methods were compared to examine their consistency. Another fifty sample sites were selected and their 
panoramic green view index obtained from GVEI and manual methods were correlated using statistic software of Origin. The results showed that the green view index values from GVEI and manual methods were highly correlated $(R=0.96$, and $\mathrm{p}<0.05)$. However, we noted that the GVEI method slightly overestimated the distributions of green vegetation (Figure 4a). The overestimation was mainly attributed to the misclassification of artificial green features in some iPhone images. However, as the manual classification method is laborious and time consuming, it is not suitable for sizeable image processing work. Considering the fact that misclassified artificial green features possessed a relative small portion of landscape in urban park, the slight overestimation results should have no much influence on the further analysis. Same situation happened to the correlation results between the panoramic green view indices calculated using iPhone and GSV images (Figure 4b), the green vegetation distribution results obtained from iPhone image and GSV showed high correlation. The overestimation may be relative to the different image capture weather conditions. The existence of shade features slightly influenced the correlation result.
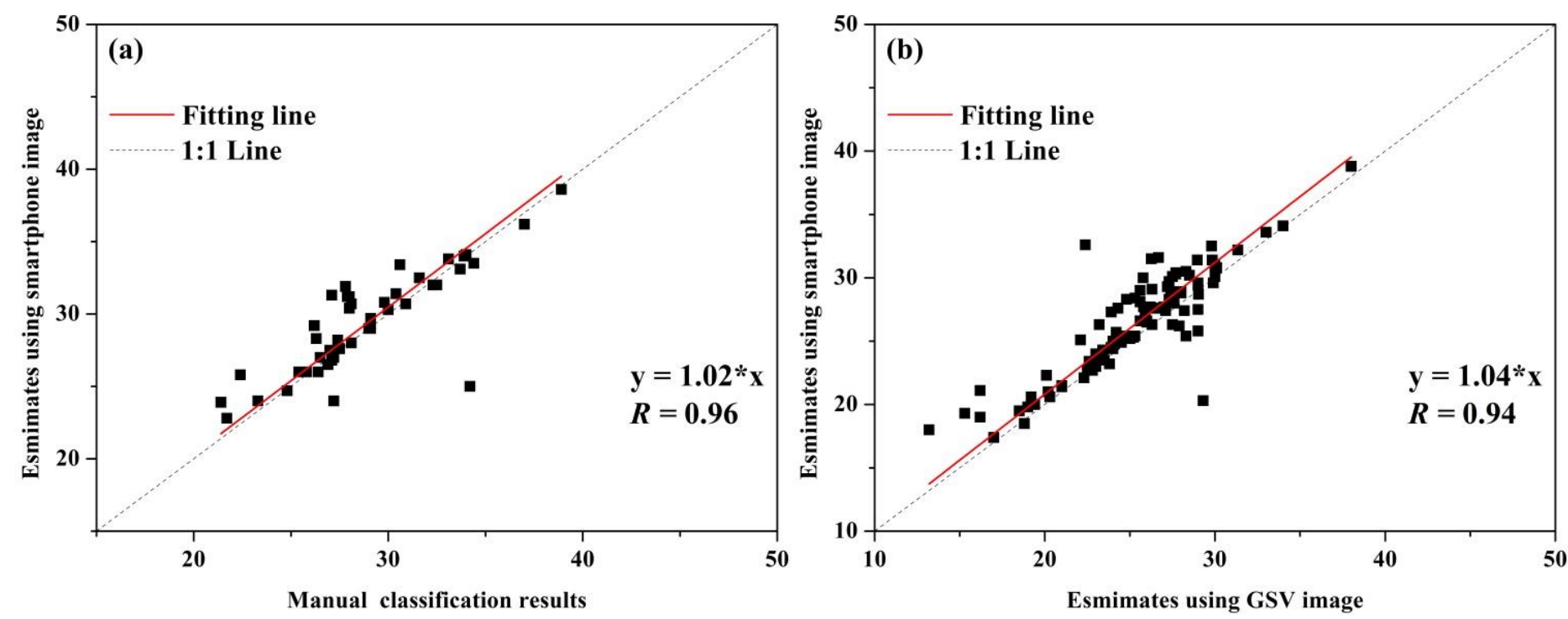

Figure 4. Linear fitting of vegetation distribution computed using estimated results using iPhone image vs. manual classification results (a) and GSV image (b).

\section{Green vegetation distribution}

The panoramic green view indices of the investigation sites were computed using the green vegetation classification dataset obtained from GVEI. The histogram statistic results were showed in Figure 5a, and the GVEI of all the study sites were spatially displayed in Figure 5b. The colors and size of dots represented the magnitude of panoramic green vegetation view index value (Eq. 2).

The histogram distribution showed that all the investigation sites possessed panoramic green view index values higher than 15\% (Figure 5a). Only seven sites were grouped into Grade 2 with green vegetation distribution rate of 15 - $20 \%$, and most of the study sites (seventy-three) occupied the vegetation distribution values that belong to Grade 3 (20 - 30\%). Using the spatial results processed based on iPhone images and GVEI method, it is easy to identify and locate the investigation sites with different greenery grades, which is indispensible for further park greenery management and planning. In the high value zone, twenty sites were classified into Grade 4 (> 30\%, Figure 5b), which meant these sites could deliver strong visual pleasure and were most beneficial to physical and mental health. Among them, there were 
fourteen sites distributed in the South Road, two high value sites in North Road, and another four sites in East Road (Figure 5b). The greenery in the West Road appears not that "green" in peoples' eyes compared to those in the other three roads.
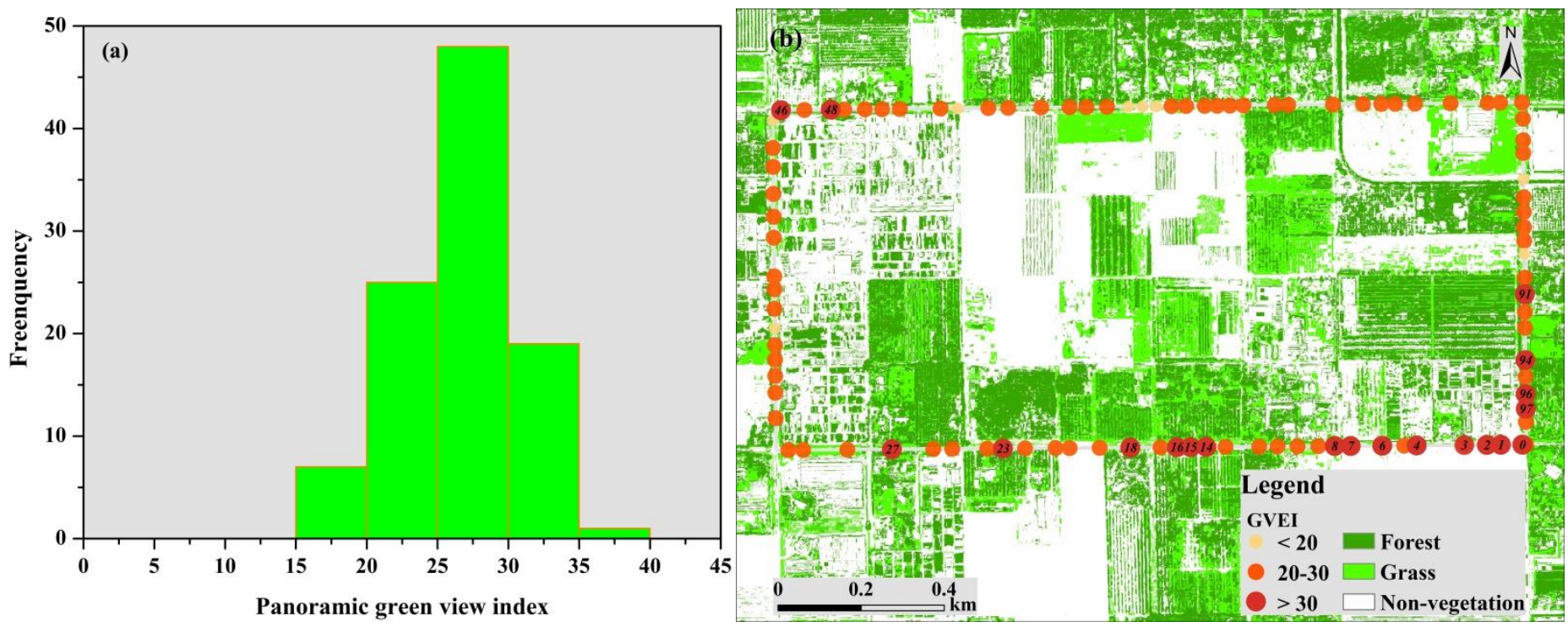

Figure 5. Vegetation distribution and statistic result obtained from GVEI method and iPhone images. (a): Histogram of the green vegetation distribution values. (b): Spatial distribution of panoramic green view index. The colors and size of dots represented the magnitude of vegetation distribution index from profile view. The results were stacked on the classification results of the RS image used in Figure 1, in which leaf green features represented trees and shrubs, light green ones were the grass areas, and white color areas were the non-vegetation features.

\section{Discussion}

This is the first study striving to examine the feasibility of typical smartphone images for monitoring and measuring the amount of park greenery that resident can see when taking physical activity, and we proposed the GVEI to help identify the green vegetation in images more accurately. To examine the usefulness of the smartphone image and the proposed index, we selected a park trail like road network, which length is a threshold that can help people improve cardiopulmonary function, strengthen body metabolism, and create a good mood and mentality [26,27]. During the data acquisition period, we spent two hours to capture panoramic images of investigation sites. Considering average walker finishes a five kilometer in 45 to 60 minutes (Healthline, https://www.healthline.com/health/exercise-fitness/average-5ktime), the whole image capturing process did not take too much extra time. Compared to other mobile image acquisition platform, e.g. unmanned aerial vehicle (UAV), smartphone is safer and more durable. It can take panoramic image at the $1.6 \mathrm{~m}$ altitude without the possibility of injury, and the battery integrated in the iPhone can support it for all day long, compared to UAV's less than half an hour battery life. Besides, given the smartphone is portable with moderate size, people can take it along when walking or jogging in the near park. They can take image of park trail following the procedure showed in this study and upload them to the mobile app such as flickr [28], which can further facilitate the park greenery analysis.

In the process of green vegetation classification, we proposed to use the GVEI method and compared the results with those based on ANNc. We found that ANNc underperformed GVEI in distinguishing green vegetation, which indicated 
that ANNc currently may not be suitable for smartphone image classification due to the absence of specific wavelength information and spectral range overlap of R/G/B bands, which underlined the practicability of GVEI method. The verification results found that the GVEI method slightly overestimated the green vegetation in the iPhone images than the manual classification results. This may be attributed to the existence of shade features in the images. Considering the labor and time intensive nature and potential subjectivity of the manual method, however, the GVEI method can be regarded as an efficient alternative especially when the image data is sizable. The semi-automatic GVEI approach combined with the manual refinement should be worth being explored to improve the efficiency and accuracy of greenery extraction in urban park. Furthermore, we also compared the results from smartphone with that based on GSV imagery. The smartphone based green view index values of the investigation sites showed high correlation with that using GSV imagery (Figure 4b). As the GSV images of 29 investigation sites were found to be obtained in 2011, the smartphone captured image can be more time-sensitive and may be used to renew the GSV computation results in the street-side, which may be an additional contribution of this study.

Despite the typical smartphone image and proposed GVEI could accurately identify park greenery information, there are some limitations that should be noted. Firstly, we used iPhone to take panoramic image of the study sites because currently more than half people in the USA use it. iPhone image is more representative in park greenery identifying and monitoring. We did not compare how much difference it is between the results from iPhone and other image capturing platforms. Secondly, influenced by the COVID-19, we could not examine the performance of smartphone image in a representative park, which is therefore a direction where further endeavors will be made in. As currently no other image data is available in park to extract green vegetation distribution patterns, smartphone panoramic image can be used as an efficient and effectively data source.

\section{Conclusions}

This study examined the feasibility of typical smartphone image used in urban park greenery study. The iPhone captured panoramic images were selected as data sources, and the GVEI method was proposed to identify the greenery in the new profile view images. The results demonstrated that the smartphone image was well suited for green vegetation extraction and the GVEI method could classify street vegetation more efficiently and accurately, compared to the manual classification and ANNc method. The investigation sites with different panoramic green view index could be located and displayed based on specific selection attributes. Further study will be conducted in representative urban parks to further examine the performance of smartphone image and GVEI.

\section{Supplementary information}

\section{Abbreviations}

GVEI: green vegetation extraction index; GSV: Google street view; NDVI: normalized difference vegetation index; NIR: near infrared; ANNc: artificial neural network classifier; UAV: unmanned aerial vehicle.

\section{Acknowledgments}

The authors wish to thank Dr. Young Gu Her in the process of data collection and manuscript writing.

\section{Authors' Contributions}




\section{Funding}

This study was supported by Open Foundation of Key Laboratory of Terrestrial Surface Pattern and Simulation, Chinese Academy of Sciences (LBKF201802). The funders had no role in study design, data collection and analysis, decision to publish, or preparation of the manuscript.

\section{Availability of data and materials}

The datasets used and analyzed during the current study are available from the corresponding author on reasonable request.

\section{Ethics approval and consent to participate}

The study was approved by the UF/IFAS Extension.

\section{Consent for publication}

Not applicable.

\section{Competing interests}

The authors declare that they have no competing interests.

\section{Author details}

${ }^{1}$ College of Resources and Environment, Shandong Agricultural University, Tai'an 271018, China. ${ }^{2}$ Key Laboratory of Terrestrial Surface Pattern and Simulation, Chinese Academy of Sciences, Bejing 100101, China. ${ }^{3}$ UF/IFAS Extension, Miami-Dade County, Homestead 33030, USA. ${ }^{4}$ Information center, Taishan Sanatorium of Shandong Province, Tai'an, China.

\section{References}

[1] Griew, P., Hillsdon, M., Foster, C., Coombes, E., Jones, A., \& Wilkinson, P. (2013). Developing and testing a street audit tool using Google Street View to measure environmental supportiveness for physical activity. International Journal of Behavioral Nutrition and Physical Activity, 10(1), 103.

[2] Hallal, P. C., Victora, C. G., Azevedo, M. R., \& Wells, J. C. (2006). Adolescent physical activity and health. Sports medicine, 36(12), 1019-1030.

[3] Gidlow, C. J., Jones, M. V., Hurst, G., Masterson, D., Clark-Carter, D., Tarvainen, M. P., ... \& Nieuwenhuijsen, M. (2016). Where to put your best foot forward: Psycho-physiological responses to walking in natural and urban environments. Journal of environmental psychology, 45, 22-29.

[4] Bang, K. S., Lee, I., Kim, S., Lim, C. S., Joh, H. K., Park, B. J., \& Song, M. K. (2017). The effects of a campus forest-walking program on undergraduate and graduate students' physical and psychological health. International journal of environmental research and public health, 14(7), 728.

[5] Lu, Y. (2019). Using Google Street View to investigate the association between street greenery and physical activity. Landscape and Urban Planning, 191, 103435. 
[6] Song, C., Ikei, H., Park, B. J., Lee, J., Kagawa, T., \& Miyazaki, Y. (2018). Psychological benefits of walking through forest areas. International journal of environmental research and public health, 15(12), 2804.

[7] Pratiwi, P. I., Xiang, Q., \& Furuya, K. (2020). Physiological and Psychological Effects of Walking in Urban Parks and Its Imagery in Different Seasons in Middle-Aged and Older Adults: Evidence from Matsudo City, Japan. Sustainability, 12(10), 4003.

[8] Cohen, D. A., Ashwood, J. S., Scott, M. M., Overton, A., Evenson, K. R., Staten, L. K., ... \& Catellier, D. (2006). Public parks and physical activity among adolescent girls. Pediatrics, 118(5), e1381-e1389.

[9] McCormack, G. R., Rock, M., Toohey, A. M., \& Hignell, D. (2010). Characteristics of urban parks associated with park use and physical activity: A review of qualitative research. Health \& place, 16(4), 712-726.

[10] Green Sight Survey Guideline Plan August 25, Osaka Osaka [EB/OL]. Japan: Osaka. (2013-08). http://www.pref.osaka.lg.jp/attach/17426/00000000/guideline.pdf.

[11] Catania, J. A., McDermott, L. J., \& Pollack, L. M. (1986). Questionnaire response bias and face $\square$ to $\square$ face interview sample bias in sexuality research.

[12] Charreire, H., Mackenbach, J. D., Ouasti, M., Lakerveld, J., Compernolle, S., Ben-Rebah, M., ... \& Oppert, J. M. (2014). Using remote sensing to define environmental characteristics related to physical activity and dietary behaviours: a systematic review (the SPOTLIGHT project). Health \& place, 25, 1-9.

[13] Li, X., Zhang, C., Li, W., Ricard, R., Meng, Q., \& Zhang, W. (2015). Assessing street-level urban greenery using Google Street View and a modified green view index. Urban Forestry \& Urban Greening, 14(3), 675-685.

[14] Aoki Yoji. The connection between the vision and the sense of greenness [J]. Gardening Magazine, 1987, 51(1): 1-10.

[15] Yu, X., Zhao, G., Chang, C., Yuan, X., \& Heng, F. (2019). Bgvi: A new index to estimate street-side greenery using baidu street view image. Forests, 10(1), 3.

[16] Yang, J., Zhao, L., Mcbride, J., \& Gong, P. Can you see green? Assessing the visibility of urban forests in cities. Landscape and Urban Planning 2009, 91(2), 97-104.

[17] Lu, Y. (2019). Using Google Street View to investigate the association between street greenery and physical activity. Landscape and Urban Planning, 191, 103435.

[18] Ye, Y., Richards, D., Lu, Y., Song, X., Zhuang, Y., Zeng, W., \& Zhong, T. (2019). Measuring daily accessed street greenery: A human-scale approach for informing better urban planning practices. Landscape and Urban Planning, 191,103434

[19] Armstrong, N., Nugent, C. D., Moore, G., \& Finlay, D. D. (2010, November). Developing smartphone applications for people with Alzheimer's disease. In Proceedings of the 10th IEEE International Conference on Information Technology and Applications in Biomedicine (pp. 1-5). IEEE.

[20] Wang, D., Xiang, Z., \& Fesenmaier, D. R. (2016). Smartphone use in everyday life and travel. Journal of travel research, 55(1), 52-63.

[21] Motohka, T., Nasahara, K. N., Oguma, H., \& Tsuchida, S. Applicability of green-red vegetation index for remote sensing of vegetation phenology. Remote Sensing 2010, 2(10), 2369-2387.

[22] Tian, F., Brandt, M., Liu, Y. Y., Verger, A., Tagesson, T., Diouf, A. A., ... \& Fensholt, R. Remote sensing of vegetation dynamics in drylands: Evaluating vegetation optical depth (VOD) using AVHRR NDVI and in situ green biomass data over West African Sahel. Remote Sensing of Environment 2016, 177, 265-276. 
[23] Ren, J. (2012). ANN vs. SVM: Which one performs better in classification of MCCs in mammogram imaging. Knowledge-Based Systems, 26, 144-153.

[24] Moraes, R., Valiati, J. F., \& Neto, W. P. G. (2013). Document-level sentiment classification: An empirical comparison between SVM and ANN. Expert Systems with Applications, 40(2), 621-633.

[25] Orihara N. Evaluation of Green Landscape-A Study on Evaluation Method of Green for Good Landscape Formation. Investigation and Research Periodical 2006, 20 (142): 4-13.

[26] Cruikshank, T. (2016). Meditate Your Weight: A 21-Day Retreat to Optimize Your Metabolism and Feel Great. Harmony.

[27] Beres, D. (2017). Whole motion: Training your brain and body for optimal health. Simon and Schuster.

[28] Song, X. P., Richards, D. R., \& Tan, P. Y. (2020). Using social media user attributes to understand humanenvironment interactions at urban parks. Scientific reports, 10(1), 1-11. 
Figures

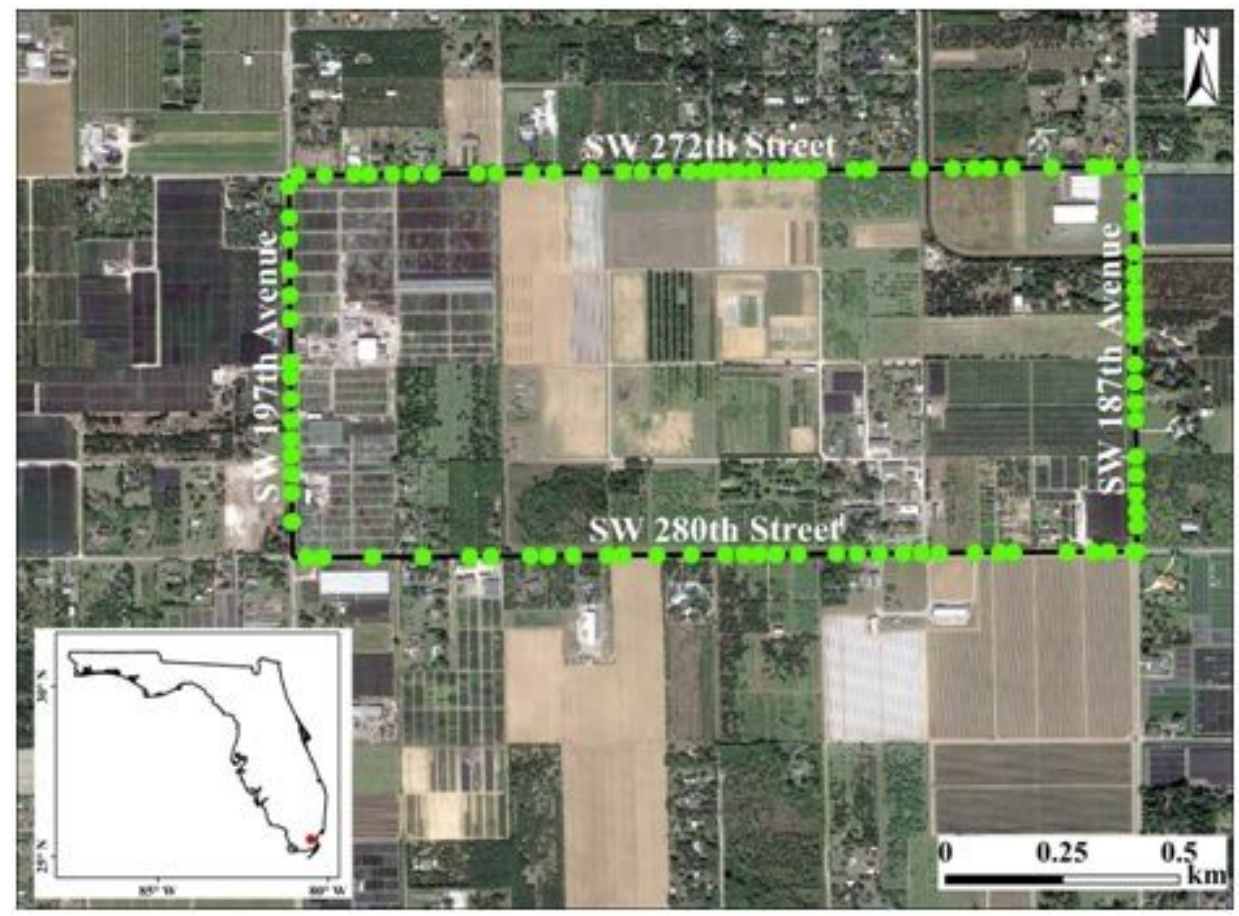

Figure 1

Location of the study region. The green dots denote the investigation sites where high quality panoramic image were obtained to extract the greenery. The background image was obtained from Google map.
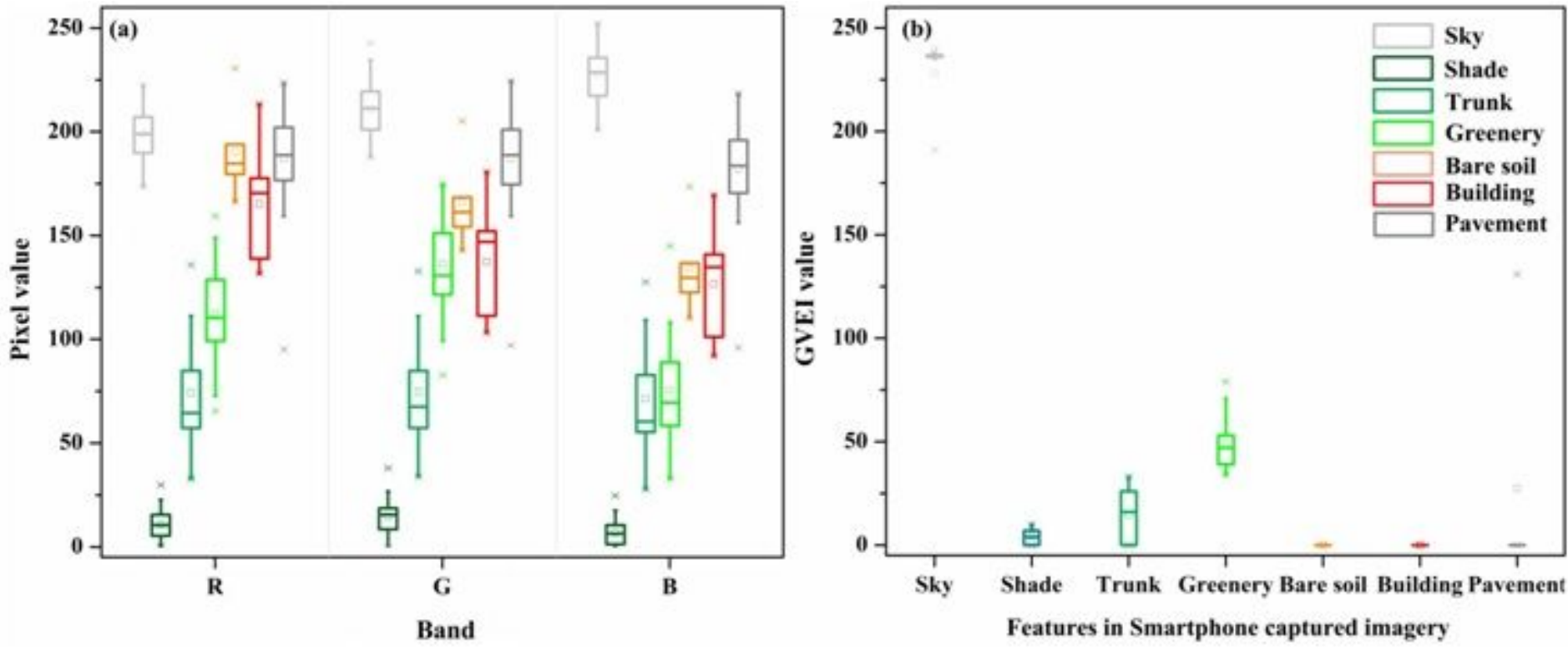

Figure 2

Pixel value and GVEI range of common features in the iPhone captured panoramic imagery. (a): Pixel value range of common features; (b): GVEI ranges of the features. 


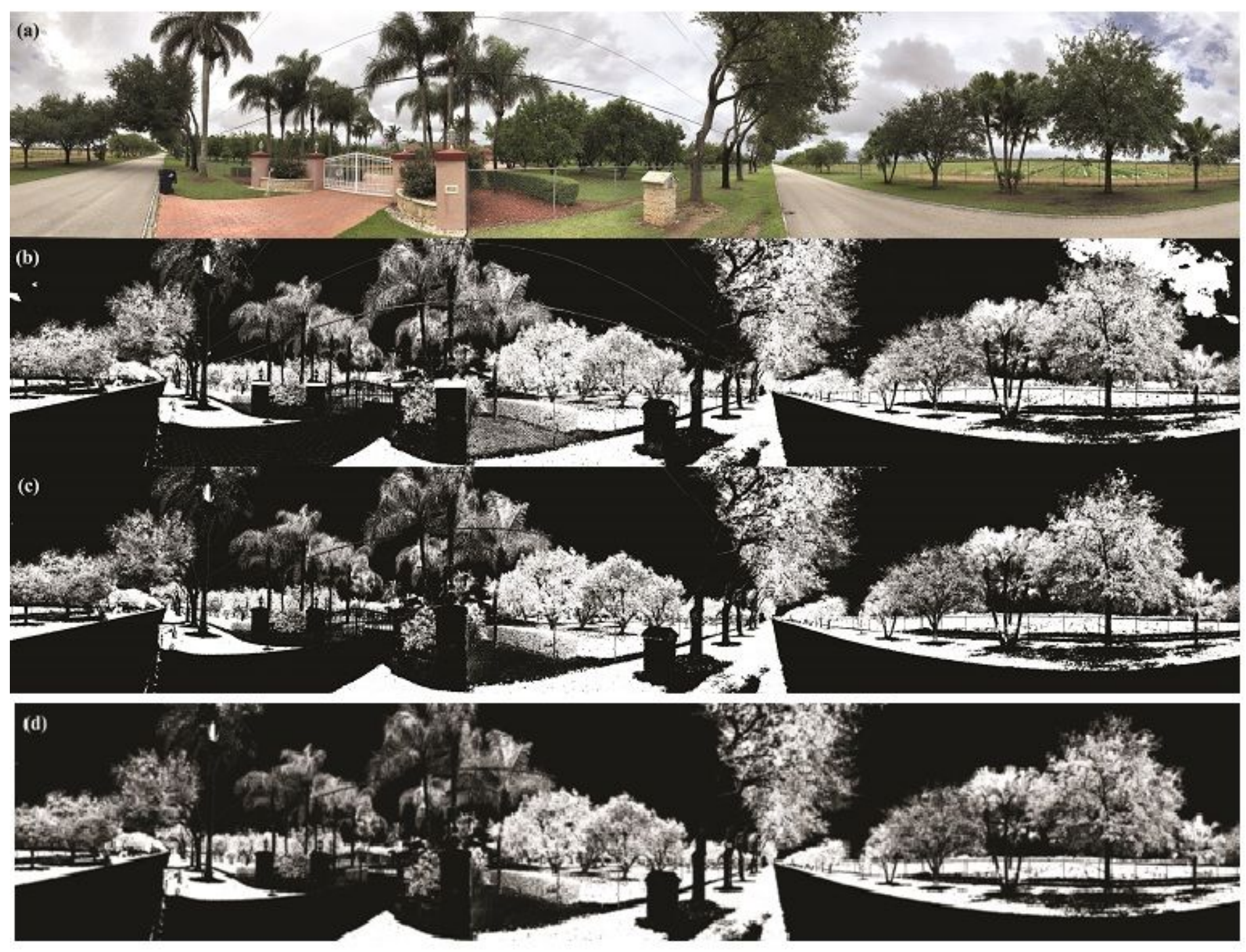

Figure 3

An example of iPhone image and green vegetation classification results. (a):Original image, (b):ANNc result, (c): GVEI processing result, and (d):manual classification. 

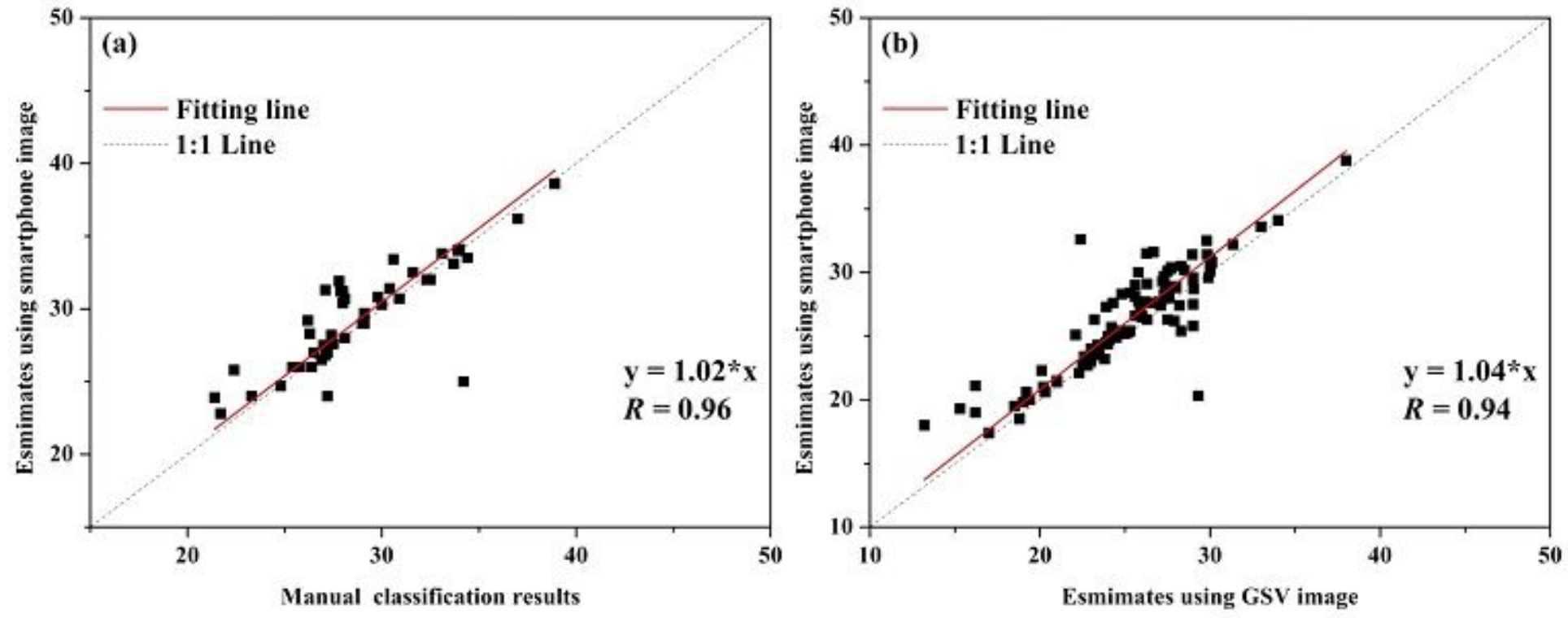

Figure 4

Linear fitting of vegetation distribution computed usingestimated results using iPhone image vs.manual classification results(a) and GSV image (b).
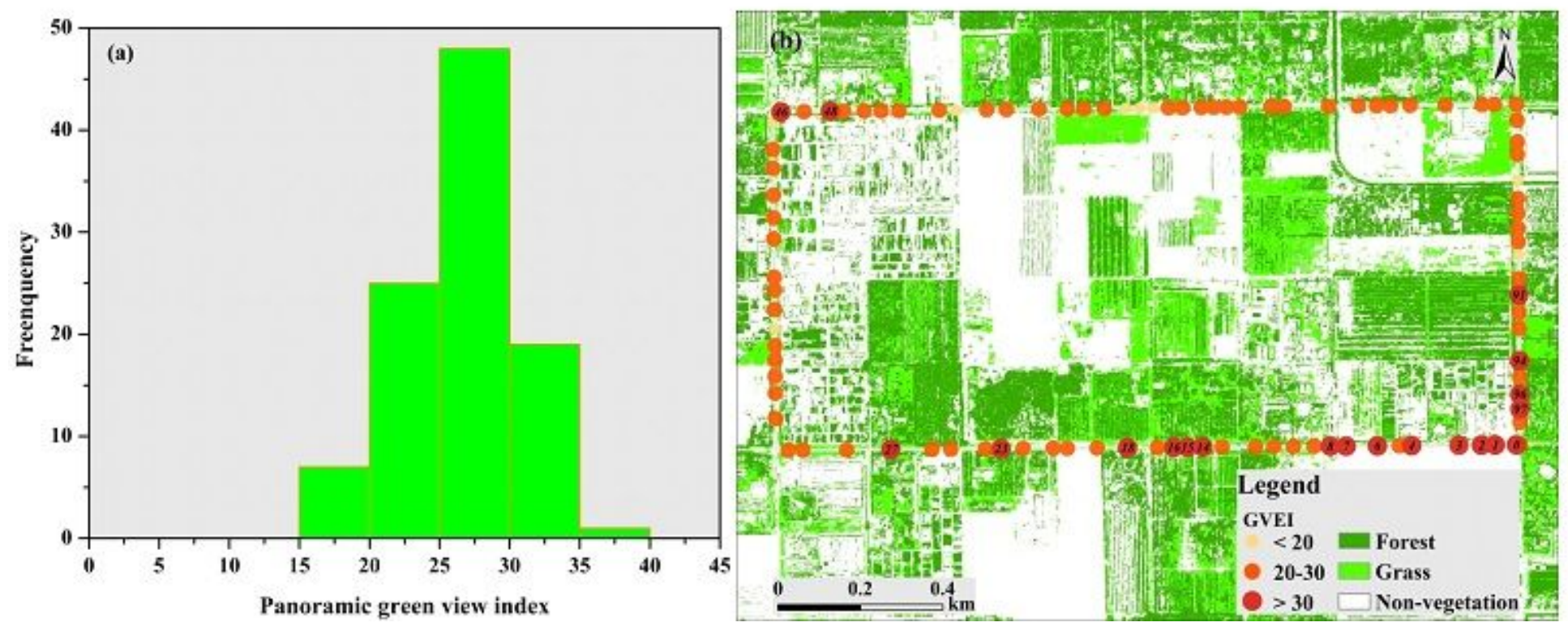

Figure 5

Vegetation distribution and statistic result obtained from GVEI method and iPhone images. (a):Histogram of the green vegetation distribution values. (b):Spatial distribution of panoramic green view index. The colors and size of dots represented the magnitude of vegetation distribution index from profile view. The results were stacked on the classification results of the RS image used in Figure 1, in which leaf green 
features represented trees and shrubs, light green ones were the grass areas, and white color areas were the non-vegetation features. 